\title{
Design Of One Plan Suspension Systems with Special Type of Double Sampling through Minimum Sum of Risks Using Spread Sheets
}

\author{
H. Shubhajyothi

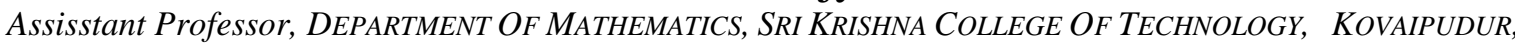 \\ COIMBATORE-42.Tamilnadu, India
}

\begin{abstract}
Acceptance sampling is concerned with inspection and decision making regarding products, one of the oldest aspects of quality assurance. The most effective of acceptance sampling is not to "inspect quality into the product" but rather as an audit tool to ensure that the output of a process conforms to requirements. Acceptance sampling is most likely to be useful in few of these cases

i) when testing is destructive

ii) when the cost of $100 \%$ inspection is extremely high

iii) when there are potentially serious product liability risks and the vendor's process is satisfactory, a program for continuously monitoring the product is necessary.

The Characteristics of "Selection of one plan suspension system with special type of double sampling originally developed by K.K.Suresh and V.Sangeetha are reconsidered in this paper from computational point of view.In this paper spread-sheet-excel work sheet and algorithm are used to calculate the probability of accepting a lot given the proportion non-conforming under one plan suspension system with special type of double sampling (STDS) plan as reference plan.
\end{abstract}

Keywords: OP, STDS, RQL, EXCEL WORKSHEET, ARL

\section{Introduction}

The effectiveness of a small sample lot-by-lot sampling system can be changed by the use of cumulative results as a basis for suspending inspection was proved by CONE and DODGE (1962).The success of this lies in the fact that the producer corrects what is wrong and submits satisfactory written evidence of action taken before inspection is resumed. The samples are chosen small so the production cost is also less.

TROXELL(1972) had incorporated a suspension rule to suspend inspection on the basis of unfavorable lot history when small sampling plans are desired. .A one-plan (OP) suspension system is a combination of a suspension rule and a single lot-by lot sampling plan. This paper relates to the procedure for the selection of OP suspension system with special type double sampling plan(STDS) as reference plan using minimum sum of risks involving products and consumer's quality levels. In this paper we have presented a method of selection of the plan using excel worksheet.

\section{Operating Procedure for STDS plan}

The Operating procedure of the STDS plan is as follows:

1 .Let the sample size $n_{1}$ be chosen at random from a lot and number of defectives be $\boldsymbol{d}_{\mathbf{1}}$.If $\boldsymbol{d}_{\mathbf{1}} \geq 0$, reject the lot. If $\boldsymbol{d}_{\mathbf{1}}=0$, select a second random sample of size $\boldsymbol{n}_{2}$.

2. If $d_{2} \leq 1$, accept the lot. Otherwise if $d_{2} \geq 2$, reject the lot.

A compact representation of the STDS plan is given in the table below:

\begin{tabular}{|c|l|l|l|}
\hline Stage & $\begin{array}{l}\text { Sample } \\
\text { size }\end{array}$ & AC & Re \\
\hline 1 & $n_{1}$ & $*$ & 1 \\
\hline 2 & $n_{2}$ & 1 & 2 \\
\hline
\end{tabular}

\section{- NO DECISION}




\section{Conditions for Application of One-plan Suspension}

$>$ Production is reasonably steady as a result the process becomes continuous.

> Samples are taken from lot substantially in the order of production so that observed variations in quality of product reflect process performance.

$>$ Inspection information is prompt since the inspection is performed closely.

Inspection is by attributes with quality measured in terms of fraction defective $\mathrm{p}$.

$>$ Either a single sample, double sample or multiple samples of sample size $\mathrm{n}$ is chosen.

\section{Operatin procedure of One-plan Suspension}

$>$ For the product under consideration establish a Reference quality level (RQL).This RQL termed as np represents the desired quality at delivery considering the needs of service and cost of production.

$>$ Consider the established RQL select a suspension system.

$>$ Apply the suspension rule to the first, second,...... th lot then to each successive group of $\mathrm{k}$ lots.

$>$ If any lot is rejected, declare the lot non-conforming and dispose it in accordance with standard procedures.

$>$ If for any lot, the suspension rule occurs, declare the current lot non-conforming and also declare the process non-conforming.

$>$ When the process is judged non-conforming

i) Notify the submitting agency that additional lots may be submitted for inspection agency that action has been taken to assure the submission of satisfactory material.

ii) Dispose the current non-conforming lot in accordance with standard procedures.

iii) When satisfactory evidence of corrective action is furnished start inspection again with next succeeding lot and with this lot begin accumulation.

iv) If it becomes necessary to refuse lot submission a second time, so advice are appropriate higher authority and notify the submitting agency that further submission will be refused until evidence satisfactory to the higher authority has been approved.

\section{Average Run Length}

According to Troxell(1972) the expected time to the suspension or average run length of the suspension rule $(\mathrm{j}, \mathrm{k})$ designated as $\mathbf{A R L}(\mathbf{j}, \mathbf{k})$ given by

i) $\operatorname{ARL}(\mathbf{j}, \mathbf{j})=\frac{1-\left(1-p_{a}\right)^{j}}{p_{a}\left(1-p_{a}\right)^{j}}, \mathrm{j} \geq 2$

ii) ARL $(\mathbf{j}, \infty)=\frac{j}{1-p_{a}} \quad$ mean of the negative binomial distribution with parameter $\mathbf{j}$.

iii)ARL $(\mathbf{2}, \mathbf{K})=\frac{2-P_{A}^{K-1}}{\left(1-P_{A}\right)\left(1-P_{A}^{K-1}\right)}, k \geq 2$.

Tables developed for the selection of the plan parameters for designing one-plan suspension system with double sampling plan as reference plan is given using excel sheets.

\section{Selection Of minimum risks One-plan Suspension system with Special Type of Double Sampling \\ Plans}

The procedure below is used for selecting plan for given $p_{1}, p_{2}, \alpha, \beta$.

1. Compute the operating ratio.

2. With the computed value of operating ratio enter table in row headed by $\frac{p_{2}}{p_{1}}$ which is equal to or just smaller than that.

3. For determining the parameter $\mathrm{k}$ and $\phi$ one proceeds from left to right in the row found in step 2 so that $\alpha, \beta$ and equal or slightly lesser. 
4. The sample size $\mathrm{n}$ is given by $\mathrm{n}=\frac{n p_{1}}{p_{1}}$ where $n p_{1}$ is given in the column heading to the two parameters $\mathrm{k}$ and $\phi$ as found previously.

\section{Construction of Tables}

The probability of accepting a lot given the proportion non-conforming under one-plan suspension system with special type double sampling (STDS) plan as reference plan is given as

$$
P_{A}(2, K)=\frac{1+P a-P_{a}^{k}}{2-p_{a}^{k-1}}
$$

Where $p_{a}=e^{-n p}(1+\phi n p)$ gives

$$
P_{A}(2, K)=\frac{1+P a-P_{a}^{k}}{2-p_{a}^{k-1}}=\frac{1+\left(e^{-n p}(1+\phi n p)-\left(e^{-n p}(1+\phi n p)\right)^{k}\right.}{2-\left(e^{-n p}(1+\phi n p)\right)^{k-1}}
$$

Steps To Calculate Minimum Sum Of Risks and the parameters K and $\phi$

1.Fixed $n p_{1} 0.05(0.10) 1.35$

2. $\frac{p_{2}}{p_{1}}$ are fixed $16(1) 44$

3. $n p_{21}=\frac{p_{2}}{p_{1}} * n p_{1}$

4. $\alpha+\beta=\left(1-p_{a}\left(p_{1}\right)\right)+p_{a}\left(p_{2}\right)$ is calculated.

5 .The values of parameters $\mathrm{k}$ and $\phi$ are obtained after step 4.

\section{Algorithm :}

To calculate probability of accepting a lot given the proportion non-conforming under one-plan suspension system with STDS plan as reference plan.

$$
\begin{aligned}
& \text { Input }: p_{1}, p_{2}, k, n, \phi \\
& \text { Output }: \alpha, \beta
\end{aligned}
$$

\section{Algorithm}

Step. $1 X \leftarrow \frac{p_{2}}{p_{1}}$

Step. 2 Calculate $n p_{2}=\mathrm{X}^{*}\left(n p_{1}\right)$

Step3. Calculate $\alpha$

$$
P_{A}(2, K)=\frac{1+P a-P_{a}^{k}}{2-p_{a}^{k-1}}
$$

Step.4 Calculate $\beta$ using

$$
P_{A}(2, K)=\frac{1+P a-P_{a}^{k}}{2-p_{a}^{k-1}}
$$




\begin{tabular}{|r|r|r|r|r|l|r|r|r|r|}
\hline p2/p1 & np1 & $\mathrm{k}$ & \multicolumn{1}{|c|}{$\phi$} & \multicolumn{1}{l|}{ np2 } & \multicolumn{1}{l|}{$\mathrm{pa}$} & $\alpha$ & $\alpha \%$ & \multicolumn{1}{|c|}{$\beta$} & $\beta \%$ \\
\hline $\mathbf{4 4}$ & $\mathbf{0 . 0 5}$ & $\mathbf{3}$ & $\mathbf{0 . 0 5}$ & $\mathbf{2 . 2}$ & $\mathbf{0 . 9 7 0 9 7 7}$ & $\mathbf{0 . 0 2 9 0 2 3}$ & $\mathbf{0 . 0 0 0 2 9}$ & $\mathbf{1 0 . 1 0 9 4 5}$ & $\mathbf{0 . 1 0 1 0 9 5}$ \\
\hline 44 & 0.05 & 3 & 0.1 & 2.2 & 0.920412 & 0.079588 & 0.000796 & 11.09448 & 0.110945 \\
\hline 44 & 0.05 & 3 & 0.15 & 2.2 & 0.820854 & 0.179146 & 0.001791 & 12.08071 & 0.120807 \\
\hline 44 & 0.05 & 3 & 0.2 & 2.2 & 0.621401 & 0.378599 & 0.003786 & 13.0679 & 0.130679 \\
\hline 44 & 0.05 & 3 & 0.25 & 2.2 & 0.164298 & 0.835702 & 0.008357 & 14.05583 & 0.140558 \\
\hline & & & & & & & & & \\
\hline
\end{tabular}

Table.1 To find the minimum sum of risks for given operating ratio and parameters $\mathrm{k}$ and $\phi$

Table. 1 clearly shows the minimum sum of risks is for the operating ratio $44, \mathrm{k}=3, \phi=0.05$.

9. Stepwise procedure to formulate table :2

Each value are predefined relationship to the other values, a change in one value needs change in other value. Spreadsheet applications are computer programs that lets us create and manipulate spreadsheets electronically.

\begin{tabular}{|c|c|c|}
\hline Cell & Expression & Formula \\
\hline A17 & Operating ratio & $\frac{p_{2}}{p_{1}}$ \\
\hline K16 & Given Value & $n p_{1}$ \\
\hline K17 & Given Value & $n p_{1} * \frac{p_{2}}{p_{1}}$ \\
\hline L17 & Parametric value & K \\
\hline L18 & & $\operatorname{Exp}\left(-n p_{1}\right)$ \\
\hline L19 & & $1+\phi n p_{1}$ \\
\hline $\mathrm{L} 20$ & & $\operatorname{Exp}\left(-n p_{1}\right) * 1+\phi n p_{1}$ \\
\hline L21 & & Power $\left(\operatorname{Exp}\left(-n p_{1}\right) * 1+\phi n p_{1}, \mathrm{~K}\right)$ \\
\hline $\mathrm{L} 22$ & & Power $\left(\operatorname{Exp}\left(-n p_{1}\right) * 1+\phi n p_{1}, \mathrm{~K}-1\right)$ \\
\hline $\mathrm{L} 23$ & & 2- Power $\left(\operatorname{Exp}\left(-n p_{1}\right) * 1+\phi n p_{1}, \mathrm{~K}-1\right)$ \\
\hline L24 & & $1+\operatorname{Exp}\left(-n p_{1}\right) * 1+\phi n p_{1}-\operatorname{Power}\left(\operatorname{Exp}\left(-n p_{1}\right)^{*} 1+\phi n p_{1}, \mathrm{~K}\right)$ \\
\hline L25 & $p_{a}=1-\alpha$ & $\begin{array}{c}1+\operatorname{Exp}\left(-n p_{1}\right)^{*} 1+\phi n p_{1}-\operatorname{Power}\left(\operatorname{Exp}\left(-n p_{1}\right)^{*} 1+\phi n p_{1}, \mathrm{~K}\right) / 2 \text { - Power } \\
\left(\operatorname{Exp}\left(-n p_{1}\right)^{*} 1+\phi n p_{1}, \mathrm{~K}-1\right)\end{array}$ \\
\hline M17 & Parametric Value & $\phi$ \\
\hline M18 & & $\operatorname{Exp}\left(-n p_{2}\right)$ \\
\hline M19 & & $1+\phi n p_{2}$ \\
\hline M20 & & $\operatorname{Exp}\left(-n p_{2}\right) * 1+\phi n p_{2}$ \\
\hline
\end{tabular}




\begin{tabular}{|c|c|c|}
\hline M21 & Power $\left(\operatorname{Exp}\left(-n p_{2}\right) * 1+\phi n p_{2}, \mathrm{~K}\right)$ \\
\hline M22 & & Power $\left(\operatorname{Exp}\left(-n p_{2}\right)^{*} 1+\phi n p_{2}, \mathrm{~K}-1\right)$ \\
\hline M23 & & $2-\operatorname{Power}\left(\operatorname{Exp}\left(-n p_{2}\right) * 1+\phi n p_{2}, \mathrm{~K}-1\right)$ \\
\hline M24 & & $1+\operatorname{Exp}\left(-n p_{2}\right)^{*} 1+\phi n p_{2}-\operatorname{Power}\left(\operatorname{Exp}\left(-n p_{2}\right)^{*} 1+\phi n p_{2}, \mathrm{~K}\right)$ \\
\hline M25 & $\beta$ & $1+\operatorname{Exp}\left(-n p_{2}\right) * 1+\phi n p_{2}-\operatorname{Power}\left(\operatorname{Exp}\left(-n p_{2}\right) * 1+\phi n p_{2}, \mathrm{~K}\right)$ \\
& $12-\operatorname{Power}\left(\operatorname{Exp}\left(-n p_{2}\right) * 1+\phi n p_{2}, \mathrm{~K}-1\right)$ \\
\hline
\end{tabular}

Example

For the given operating ratio $\frac{p_{2}}{p_{1}}=43$ and also given value of $n p$

Cell "K 28" gives the value of $n p_{2}=2.15$

Cell " $\mathrm{L} 28$ " is the value of $\mathrm{K}=3$

Cell "L 29" gives the value $\operatorname{Exp}\left(-n p_{1}\right)=0.9512294$

Cell "L 30 " gives the value of $1+\phi n p_{1}=1.0025$

Cell "L 31" gives the value $\operatorname{Exp}\left(-n p_{1}\right) * 1+\phi n p_{1}=0.9536$

Cell "L 32" gives the value Power( $\left.\operatorname{Exp}\left(-n p_{1}\right) * 1+\phi n p_{1}, \mathrm{~K}\right)=0.8671$

Cell "L 33" gives the value Power $\left(\operatorname{Exp}\left(-n p_{1}\right) * 1+\phi n p_{1}, \mathrm{~K}-1\right)=0.90936$

Cell "L 34" gives the value 2- Power $\left(\operatorname{Exp}\left(-n p_{2}\right) * 1+\phi n p_{2}, \mathrm{~K}-1\right)=1.0906327$

Cell "L 35" gives the value $1+\operatorname{Exp}\left(-n p_{1}\right) * 1+\phi n p_{1}-\operatorname{Power}\left(\operatorname{Exp}\left(-n p_{1}\right) * 1+\phi n p_{1}, \mathrm{~K}\right)=1.0864281$

Cell "L 36 " gives the value of $1+\operatorname{Exp}\left(-n p_{1}\right) * 1+\phi n p_{1}$ - Power( $\left.\operatorname{Exp}\left(-n p_{1}\right) * 1+\phi n p_{1}, \mathrm{~K}\right) / 2$ - Power

$$
\left(\operatorname{Exp}\left(-n p_{1}\right)^{*} 1+\phi n p_{1}, \mathrm{~K}-1\right)=0.9961447
$$

Cell "L 37" gives the value of $\alpha=0.003$

Similarly for $\beta$

By following the steps given above the table: 2 is manipulated.

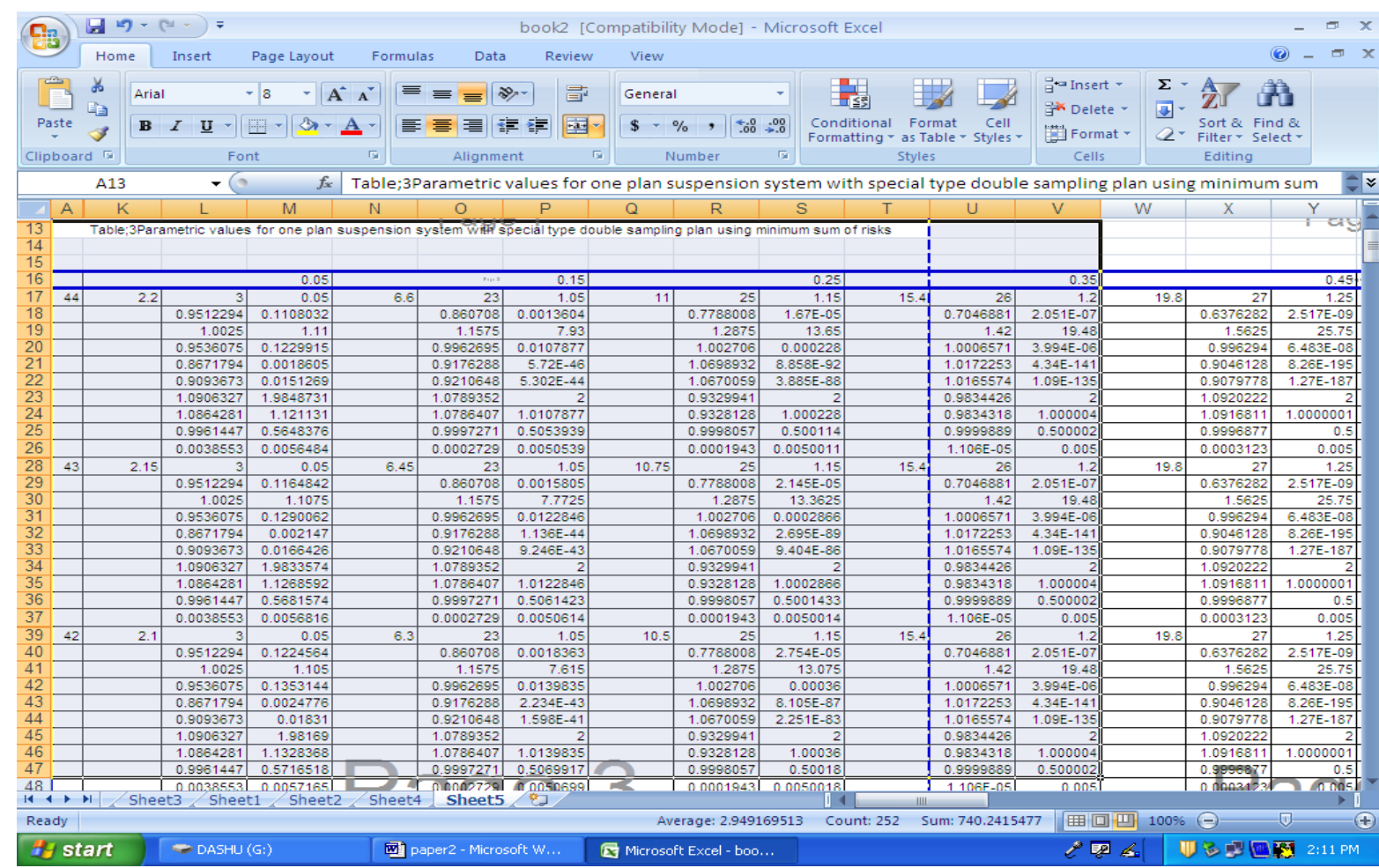




\section{Conclusion}

Ms-Excel is a window based electronic spreadsheet used to perform statistical and mathematical calculations easily. It is a component of Microsoft office. It is very powerful and since it has a Graphical user interface it is simple to learn and view graphs. The major use of excel sheet is built-in functions. Functions are special purpose programs that accept data and return a value after performing calculations on the data. It is more efficient way of performing mathematical operations than formulas. The tables are useful for both producer and consumer for obtaining good quality products with less cost of inspection a which can be easily evaluated using the excel sheets.

\section{References:}

[1] Cone.A.F and Dodge H.F (1962): A Cumulative results plan for small Inspection , Sandia Corporation Repring, SCR-678, ALBUQUERQUE,NM.

[2] Glouba.A (1953) :Designing single sampling inspection plans when the sample size is fixed Journal of American Statistical Association,vol48,pp278-288

[3] Lily Christina. A (1995): Contributions to the study of Design and analysis of suspension system and some other sampling plans, Ph...D Thesis, Department of Statistics, Bharathiyar University ,coimbatore-46,India.

[4] Soundararajan, v (1981):Sampling Inspection plans when the sample size is fixed ,Journal of Madras University, Section B vol44,pp91-99.

[5] Suresh K.K and Jayalakshmi .S(2007): Designing of switching system with special type double sampling plans specified quality levels, Impact Journal of Science and Technology,vol1,No1-2,pp441-49

[6] Suresh K.K and Kaviyarasu.V(2008): Certain results and tables relating Qss-1 with conditional RGS plan, IAPQR Transaction, vol.1No1-2pp61-70.

[7] Suresh K.K. and Saminathan .R(2007): Selection of Multiple repetitive group sampling plan involving Maximum Allowable percent Defective and Maximum Allowable Average Outgoing Quality, International Journal of Statistics and Management system,Vol.2No12pp22-30.

[8] Vijayaraghavan.R(1989)On Designing Multiple deferred state sampling (Mds-1(0,2)) plans involving minimum sum of risks ,Journal of Applied statistics,vol16,No1,pp87-88.

[9] Troxell.J.R(1972) : An investigation of Suspension system for small inspection, Ph. D Thesis, Rutgers University, New Jersey.

[10. Troxell,J.R(1980): Suspension system for small inspection,Technometrics,Vol.22 No.14,pp517-533.

\begin{tabular}{|c|c|c|c|c|c|c|c|c|c|c|c|c|c|c|c|c|c|c|c|c|c|c|c|c|c|c|c|c|}
\hline & & 0.05 & & 0.15 & & 0.25 & & 35 & & 0.45 & & 0.55 & & 0.65 & & 0.75 & & 0.85 & & 0.95 & & 1.05 & & 1.15 & & 1.25 & & 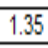 \\
\hline \multirow[t]{2}{*}{44} & 3 & 0.1 & 23 & 1.1 & 25 & 1.2 & 26 & & 27 & 1.25 & 29 & 1.35 & 30 & 1.4 & 30 & 1.4 & 30 & 1.4 & 30 & 1.4 & 30 & 1.4 & 30 & 1.4 & 30 & 1.4 & 30 & 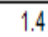 \\
\hline & 0 & 0 & & 0 & & 0 & & & & 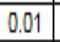 & & 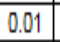 & & 0.01 & 0.01 & 0.01 & 0.03 & 01 & & 0.01 & 1 & 0.01 & 0.1 & 01 & 0.1 & 01 & \begin{tabular}{|l|}
0.1 \\
\end{tabular} & 0.01 \\
\hline \multirow[t]{2}{*}{43} & 3 & 0.1 & 23 & 11 & 25 & 1.2 & 26 & & 27 & 1.25 & 29 & 1.35 & 30 & 14 & 30 & 14 & 30 & 14 & 30 & 14 & 30 & 4 & 30 & 4 & 30 & 14 & 30 & $\overline{14}$ \\
\hline & 0 & 0 & 0 & . & 0 & 01 & 0 & 0 & 0 & 0.01 & & 0.01 & 0 & 0.01 & 0.01 & 0.01 & \begin{tabular}{|l|}
0.03 \\
\end{tabular} & 0.01 & 0 & 0.01 & 0.1 & 0.01 & 0.1 & 0.01 & 0.1 & 0.01 & 0.1 & 0.01 \\
\hline \multirow[t]{2}{*}{42} & 3 & 0.1 & 23 & 1.1 & 25 & 1.2 & 26 & 1 & 27 & 1.25 & 29 & 1.35 & 30 & 1.4 & 30 & 1.4 & 30 & 1.4 & 30 & 1.4 & 30 & 1.4 & 30 & 1.4 & 30 & 1.4 & 30 & 1.4 \\
\hline & 0 & 0 & 0 & 0 & 0 & 4 & 1 & 0 & 1 & 0.01 & 0 & 0.01 & 0 & 0.01 & 0.01 & 0.01 & \begin{tabular}{|l|}
0.03 \\
\end{tabular} & 0.01 & 0 & 0.01 & 0.1 & 0.01 & 0.1 & 0.01 & 0.1 & .01 & 0.1 & 0.01 \\
\hline \multirow[t]{3}{*}{41} & 3 & 0.1 & 23 & 1.1 & 25 & 1.2 & 26 & & 27 & 1.25 & 29 & 1.35 & 30 & 1.4 & 30 & 1.4 & 30 & \begin{tabular}{|l|}
1.4 \\
\end{tabular} & 30 & 1.4 & 30 & 1.4 & 30 & 1.4 & 30 & 1.4 & 30 & 1.4 \\
\hline & 0.95 & 0.1 & 0.86 & II & 0.78 & T & 0.7 & 0 & 0.64 & Tt & 0.58 & 0 & \begin{tabular}{l|}
0.5 \\
\end{tabular} & 0 & 0.47 & 0 & $\overline{0.43}$ & 0 & 0.4 & 0 & 0.3 & 0 & $\begin{array}{ll}0.3 \\
\end{array}$ & 0 & 0.3 & 01 & 31 & \\
\hline & 0 & 0 & 0 & 8 & 0 & 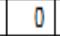 & 0 & 0 & 0 & 0.01 & 0 & 0.01 & 0 & 0.01 & 0.01 & \begin{tabular}{|l|l|} 
\\
\end{tabular} & 0.03 & 0.01 & 0 & 0.01 & 0.1 & 0.01 & 0.1 & 0.01 & 0.1 & 0.01 & 0.1 & 0.01 \\
\hline \multirow[t]{2}{*}{40} & 3 & 0.1 & 23 & 11 & 25 & 1.2 & 26 & 1 & 27 & 125 & 29 & 1.35 & 30 & 14 & 30 & 1.4 & 30 & 4 & 30 & 1.4 & 30 & 14 & 30 & 14 & 7 & 1.4 & & 14 \\
\hline & 0 & 0 & $\overline{0}$ & 0 & $\overline{0}$ & 0 & 0 & 0 & 0 & 0.01 & 0 & 0.01 & 0 & 0.01 & 0.01 & 0.01 & \begin{tabular}{|l|}
0.03 \\
\end{tabular} & 0.01 & $2 t$ & 0.01 & \begin{tabular}{|l|} 
\\
\end{tabular} & 0.01 & 0.1 & 0.01 & \begin{tabular}{l|l}
0.1 \\
\end{tabular} & 0.01 & 0.1 & 0.01 \\
\hline \multirow[t]{3}{*}{39} & 3 & 0.1 & 23 & 1.1 & 25 & 1.2 & 26 & $\sqrt{1}$ & 27 & 1.25 & 29 & 1.35 & 30 & 1.4 & 30 & 1.4 & 30 & 1.4 & 30 & 1.4 & 30 & 1.4 & 30 & 1.4 & 30 & 1.4 & 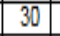 & 1.4 \\
\hline & 0.95 & 0.1 & 0.86 & 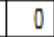 & 0.78 & 1 & 0.7 & 0 & 0.64 & 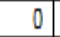 & 0.58 & 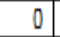 & 0.5 & 0 & 0.47 & 0 & \begin{tabular}{|l|l|} 
\\
\end{tabular} & 0 & 0.4 & 0 & 0.3 & 0 & 0.3 & 0 & \begin{tabular}{l|l}
0.3 \\
\end{tabular} & & 1 & \\
\hline & 0 & 0 & & $\pi$ & 0 & t & 0 & 0 & t & 0.01 & t & 0.01 & 0 & 0.01 & 0.01 & \begin{tabular}{|l|}
0.01 \\
\end{tabular} & \begin{tabular}{|l|}
0.03 \\
\end{tabular} & \begin{tabular}{|l|}
0.01 \\
\end{tabular} & 0 & 0.01 & 0.1 & 0.01 & 0.1 & 0.01 & $\Rightarrow$ & 0.01 & 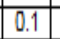 & 0.01 \\
\hline \multirow[t]{2}{*}{38} & 3 & 0.1 & 23 & 1.1 & 25 & 1.2 & 26 & 4 & 27 & 125 & 29 & 1.35 & 30 & 1.4 & 30 & 1.4 & 30 & 1.4 & 30 & 1.4 & 30 & 1.4 & 30 & 1.4 & 30 & 1.4 & 30 & 1.4 \\
\hline & 0 & 0 & 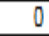 & 0 & 0 & 0 & $\pi$ & 0 & 0 & 0.01 & 0 & 0.01 & 0 & 0.01 & 0.01 & 0.01 & 0.03 & 0.01 & 0 & 0.01 & 0.1 & 0.01 & 0.1 & 0.01 & 0.1 & 0.01 & \begin{tabular}{|l|}
0.1 \\
\end{tabular} & 0.01 \\
\hline \multirow[t]{3}{*}{37} & 3 & 0. & 23 & 1.1 & 25 & 1.2 & 26 & & 27 & 1.25 & 29 & 1.35 & 30 & 1.4 & 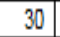 & 1.4 & 301 & 1.4 & 301 & 1.4 & 30 & 1.4 & 30 & 1.4 & 30 & 1.4 & & 1.4 \\
\hline & 0.95 & 0.2 & & 0 & 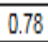 & 0 & 0.7 & 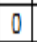 & & 0 & & & 0.5 & & & 0 & \begin{tabular}{|l|l|}
0.43 \\
\end{tabular} & & 0.4 & 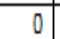 &. & & 0.0 & & & & & \\
\hline & 0 & 0 & & 0 & & 0 & 50 & $0 t$ & & 0.01 & 0 & 0.01 & 0 & 0.01 & 0.01 & 0.01 & 0.03 & 0.01 & 0 & 0.01 & 0.1 & 0.01 & 0.1 & 0.01 & 0.1 & 0.01 & 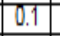 & 0.01 \\
\hline \multirow[t]{2}{*}{$\overline{36}$} & 3 & 0.1 & 23 & 1.1 & 25 & 1.2 & 26 & 1 & 27 & 1.25 & 29 & 1.35 & 30 & 1.4 & 30 & 1.4 & 30 & 1.4 & 30 & 1.4 & 30 & 1.4 & 30 & 1.4 & 30 & 1.4 & 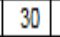 & 1.4 \\
\hline & 0 & 0 & 0 & 0 & & 0 & 0 & 0 & & 0.01 & & 0.0 & 0 & 0.01 & 0.01 & 0.01 & 0.03 & 0.01 & 0 & 0.01 & 0.1 & 0.01 & 0.1 & 0.01 & 0.1 & 0.01 & 0.1 & 0.01 \\
\hline \multirow[t]{3}{*}{35} & 3 & 0.1 & 23 & 1.1 & 25 & 1.2 & 26 & & 27 & 1.25 & 29 & 1.35 & 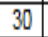 & 1.4 & (1 & 1.4 & 30 & 1.4 & 3 & 1.4 & 30 & 1.4 & 30 & 1.4 & 30 & 1.4 & t & 1.4 \\
\hline & 0.95 & 0.2 & & 0 & 0 & 0 & 0.7 & 0 & $0+1$ & 0 & & & 0.5 & 4 & & 0 & & 0 & 0.4 & 0 & 0. & 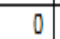 & 0.3 & & & & & \\
\hline & 0 & 0 & $\overline{0}$ & 0 & $\overline{0}$ & 0 & $\pi$ & 4 & 0 & 0.01 & 0 & 0.01 & 0 & 0.01 & 0.01 & \begin{tabular}{|l|l|}
0.01 \\
\end{tabular} & \begin{tabular}{|l|}
0.03 \\
\end{tabular} & 0.01 & 0 & 0.01 & 0.1 & 0.01 & 0.1 & 0.01 & 0.1 & 0.01 & 0.1 & 0.01 \\
\hline \multirow[t]{2}{*}{34} & 3 & 0.1 & 23 & 1.1 & 25 & 1.2 & 26 & 1 & 27 & 1.25 & 29 & 1.35 & 30 & 1.4 & 30 & 1.4 & 30 & 1.4 & 30 & 1.4 & 30 & 1.4 & 30 & 1.4 & 30 & 1.4 & 30 & 1.4 \\
\hline & 0 & 0 & 0 & 0 & 0 & 0 & 0 & & 0 & 0.0 & & 0.01 & 0 & 0.01 & 0.01 & 0.01 & 0.03 & 0.01 & 0 & 0.01 & 0.1 & 0.01 & 0.1 & 0.01 & 0.1 & 0.01 & 0.1 & 0.01 \\
\hline \multirow[t]{2}{*}{33} & \begin{tabular}{|l|}
3 \\
\end{tabular} & 0.1 & 23 & 1.1 & 25 & 1.2 & 26 & & 27 & 1.25 & 29 & 1.35 & 30 & 1.4 & 30 & 1.4 & 30 & 1.4 & 30 & 1.4 & 30 & 1.4 & 30 & 1.4 & 30 & 1.4 & 30 & 1.4 \\
\hline & 0 & 0 & $\overline{0}$ & $\pi$ & 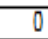 & t & 0 & 0 & 0 & 0.01 & 0 & 0.01 & 0 & 0.01 & 0.01 & \begin{tabular}{|l|}
0.01 \\
\end{tabular} & \begin{tabular}{|l|}
0.03 \\
\end{tabular} & \begin{tabular}{|l|}
0.01 \\
\end{tabular} & 0 & 0.01 & 0.1 & 0.01 & 0.1 & 0.01 & \begin{tabular}{|l|}
0.1 \\
\end{tabular} & 0.01 & \begin{tabular}{|l|}
0.1 \\
\end{tabular} & 0.01 \\
\hline \multirow[t]{3}{*}{32} & 3 & 0. & 23 & 1.1 & 25 & 1.2 & 26 & & 27 & 1.25 & 29 & 1.35 & 30 & 1.4 & 30 & 1.4 & 30 & 1.4 & 30 & 1.4 & 30 & 1.4 & 30 & 1.4 & 30 & 1.4 & 30 & 1.4 \\
\hline & 0.95 & 0.2 & 86 & 0 & 0.78 & 0 & 0.7 & 0 & 0.64 & 0 & 0.58 & 0 & 0.5 & 0 & 0.47 & 0 & \begin{tabular}{|l|l|}
0.43 \\
\end{tabular} & 0 & \begin{tabular}{|l|}
0.4 \\
\end{tabular} & 0 & 0.3 & 0 & $\begin{array}{ll}0.3 \\
\end{array}$ & 1 & 0.3 & 0 & 0.3 & 0 \\
\hline & 0 & 0 & 0 & 0 & $\overline{0}$ & 0 & 0 & 0 & 0 & 0.01 & 0 & 0.01 & 0 & 0.01 & 0.01 & 0.01 & 0.03 & 0.01 & 0 & 0.01 & 0.1 & 0.01 & 0.1 & 0.01 & \begin{tabular}{|l|}
0.1 \\
\end{tabular} & 0.01 & \begin{tabular}{|l|}
0.1 \\
\end{tabular} & 0.01 \\
\hline \multirow[t]{2}{*}{31} & 3 & 0.1 & 23 & 1.1 & 25 & 1.2 & 26 & $F$ & \begin{tabular}{|l|}
27 \\
\end{tabular} & 1.25 & 29 & 1.35 & 30 & 1.4 & 30 & 1.4 & 30 & 1.4 & 30 & 1.4 & 30 & 1.4 & 30 & 1.4 & 30 & 1.4 & 30 & 1.4 \\
\hline & 0 & 0 & 0 & 0 & 0 & 0 & 0 & 0 & 0 & 0.01 & 0 & 0.01 & 0 & 0.01 & 0.01 & 0.01 & \begin{tabular}{|l|}
0.03 \\
\end{tabular} & 0.01 & 0 & 0.01 & 0.1 & 0.01 & 0.1 & 0.01 & 0.1 & 0.01 & 0.1 & 0.01 \\
\hline \multirow[t]{2}{*}{30} & 3 & 0.1 & 23 & 1.1 & 25 & 1.2 & 26 & 1 & 27 & 1.25 & 29 & 1.35 & 30 & 1.4 & 30 & 1.4 & 30 & 1.4 & 30 & 1.4 & 30 & 1.4 & 30 & 1.4 & 30 & 1.4 & 30 & 1.4 \\
\hline & 0 & 0 & 0 & 0 & 0 & 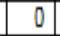 & 0 & 0 & 0 & 0.01 & 0 & 0.01 & 0 & 0.01 & 0.01 & 0.01 & 0.03 & 0.01 & 0 & 0.01 & 0.1 & 0.01 & 0.1 & 0.01 & 0.1 & 0.01 & 0.1 & 0.01 \\
\hline
\end{tabular}




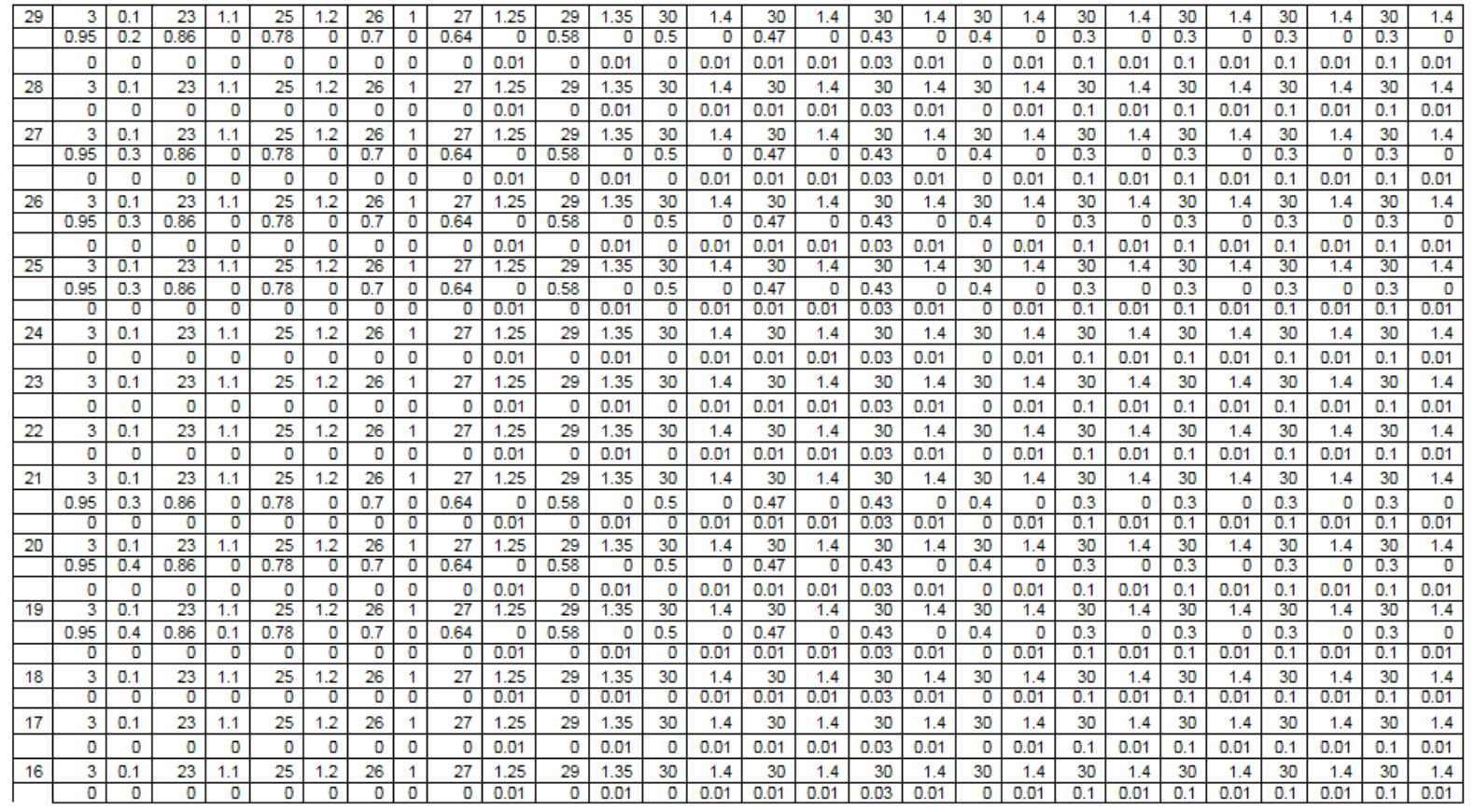

Table.2 Parametric values for one plan suspension system with special type double sampling plan using minimum sum of risks 